\title{
Healthy by choice
}

\section{How can physical scientists contribute to biomedical applications and healthcare?}

The Nobel Prizes in Medicine won by scientists such as Francis Crick, Tadeusz Reichstein, Paul Lauterbur and Peter Mansfield are a clear demonstration that some of the most important innovations in medical technology come from the ideas and efforts of scientists with a physical sciences background. The interest of physicists, chemists and materials scientists for life sciences and medical applications in particular has always been there, but there have never been as many opportunities in these fields as there are today.

In this issue we publish two articles exploring the motivations for some physical scientists to move away from their original research field and use their background to contribute to the development of biomedical applications, as well as the reasons why some companies traditionally operating in the consumer electronics sector expand into healthcare.

Strategic decisions in companies are inevitably driven by economics. And the healthcare market is expanding, as highlighted by Hans Hofstraat of Philips in his interview ${ }^{1}$. Society is ageing and there is increasing need for more user-friendly medical technologies, as well as cheaper and faster diagnostic tools. At the same time we have a deeper understanding of the molecular origin of illnesses and ways of treating them. These factors generate a wide range of opportunities for companies with a physical sciences background. Accordingly, the research programme at Philips has recently been restructured, affecting the work of most of the scientists previously working on more traditional technological themes - such as information storage.

An academic's point of view is provided by Younan Xia, in his commentary on nanomedicine ${ }^{2}$. Based closely on his own

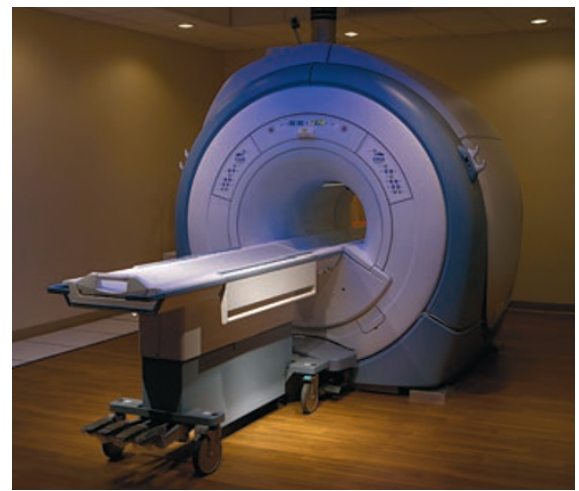

multidisciplinary programmes aimed at solving current societal needs. For example, the Cooperation programme of the European Union FP7 includes health, energy, environment - and therefore climate change - and security among its thematic areas ${ }^{3}$. The United Kingdom has recently seen the start of cross-council programmes for funding multidisciplinary collaborations. After the recent restructuring of the EPSRC (Engineering and Physical Sciences Research Council), one of its four mission programmes is Towards Next-Generation Healthcare, which is included as part of a crosscouncil collaboration on Lifelong Health and Wellbeing 4 .

There is no doubt that scientific research revolutionary medical application that originated from fundamental studies.

experience, the author explains how, after the development of novel nanostructures in the past ten to fifteen years, it has become clear that these offer interesting possibilities in biomedical imaging, contrast enhancement, drug delivery and more. Immense opportunities have arisen for material scientists who can now use their knowledge to optimise the properties of these structures and improve their biocompatibility, as well as developing new structures with specific applications in mind. On the other hand, the life sciences in general are becoming more quantitative and exact, and they can benefit substantially both from the expertise of physical scientists in characterization techniques and from their quantitative approach to science.

All in all, the rationale for industry and academia to focus on biomedical applications and healthcare is obvious. These types of transitions are aided by the worldwide trend of funding should reflect the changes and needs of our society. But we agree with Younan Xia's warning concerning the concentration of resources on applicative projects - not only those associated with health - if this means decreasing the possibility of performing fundamental studies. For many scientists working in industry the changes in research programmes imposed by their company may be a positive challenge. But such decisions inevitably produce dissatisfaction in others. More generally, it should be recognized that most successful technological innovations originate from fundamental studies. For example, magnetic resonance imaging stems from nuclear magnetic resonance, which was developed for studying the structures of molecules at the atomic scale. In the long run, reducing funding for this type of research will inevitably also affect applications.

\section{References}

1. Nature Mater. 7, 761-762 (2008)

2. Xia, Y. Nature Mater. 7, 758-760 (2008).

3. http://ec.europa.eu/research/fp7

4. http://www.epsrc.ac.uk/ResearchFunding/Programmes/ Healthcare 